Re-submitted to: Journal of Membrane Sciences (JMS-08786)

Date: $\quad 26$ August 2008

\title{
Visualisation and Characterisation of Biopolymer Clusters in a Submerged Membrane Bioreactor
}

\author{
Fei-yun Sun, Xiao-mao Wang+, Xiao-yan Li*
}

Environmental Engineering Research Centre, Department of Civil Engineering, The University of Hong Kong, Pokfulam Road, Hong Kong, China

$\begin{array}{lll}\text { *Corresponding Author: } & \text { Phone: } & (852) 2859-2659 \\ & \text { Fax: } & (852) 2559-5337 \\ & \text { Email: } & \text { xlia@hkucc.hku.hk } \\ & \text { Homepage: } & \text { http://web.hku.hk/ xlia/ }\end{array}$

+Present address: School of Civil and Environmental Engineering, University of New South Wales, Sydney NSW 2052, Australia

Running Head: BPC on MBR fouling 


\begin{abstract}
A laboratory wastewater treatment membrane bioreactor (MBR) with a submerged hollow-fibre membrane was used to investigate the major foulants in sludge mixtures. Confocal laser scanning microscopy (CLSM) with a triple fluorescent staining protocol, i.e., SYTO9 for microbial cells, ConA-TRITC lectin for polysaccharides and NanoOrange for proteins, was utilised to visualise the fouling materials. A pool of biopolymer clusters (BPC) ranging from 2.5 to $60 \mu \mathrm{m}$ in size was identified in the liquid phase of the MBR sludge and in the cake sludge on the membrane surface. According to the CLSM examination, BPC are free and independent organic solutes that are different from biomass flocs and extracellular polymeric substances (EPS) and much larger than soluble microbial products (SMP). Compared to EPS, BPC contain more polysaccharides and proteins and less humic substances. It is believed that BPC are an important foulant that interacts with biomass flocs to form the sludge fouling layer on the membrane. A filtration test observed with the CLSM shows that BPC are apparently formed by the adsorption and affinity clustering of SMP within the sludge deposited on the membrane surface. The cake sludge on the fouled membrane has a much higher BPC content (16.8 mg TOC/g SS) than the MBR bulk sludge $(0.4 \mathrm{mg} \mathrm{TOC} / \mathrm{g}$ SS). It is argued that BPC behave as a glue to facilitate the growth of an impermeable sludge cake on the membrane surface, thus resulting in serious MBR fouling. These CLSM findings provide the first direct evidence of the presence of BPC in MBR and illustrate their essential role in membrane fouling.
\end{abstract}

Keywords: Biological wastewater treatment; Biopolymer cluster (BPC); Confocal laser scanning microscopy (CLSM); Membrane bioreactor (MBR); Membrane fouling. 


\section{Introduction}

Membrane bioreactor (MBR) technology has become increasingly attractive for biological wastewater treatment in recent years due to its high-quality effluent, compacted system and low sludge yield $[1,2]$. However, membrane fouling is still the major concern in wider and large-scale MBR applications [3,4]. Membrane fouling in MBR results from the deposition of biomass and other organic and inorganic foulants onto the membrane surface [1,5-7]. This fouling causes a great reduction in filtration flux and hence a large decrease in the cost-effectiveness of the treatment system $[4,8]$. Because of the complexity and variability of the foulants and their mutual interactions in the MBR, membrane fouling is a highly complex phenomenon that is still poorly understood $[9,10]$. The concentrated biomass sludge in the reactor is supposed to be the main material that forms the fouling layer on the membrane surface $[5,7,10]$. However, other organic substances in the sludge suspension, including soluble microbial products (SMP) and extracellular polymeric substances (EPS), are also believed to play an important role in the membrane fouling process [11-13].

Recent studies have revealed a pool of organic matter deemed to be biopolymer clusters (BPC) in the liquid phase of the MBR sludge mixture $[13,14]$. These BPC are neither microbial mass nor EPS, and they are non-filterable organics much larger than SMP. It is expected that BPC play a crucial role in the formation of the sludge fouling layer on the membrane surface. The accumulation of BPC within the pores of the sludge cake layer on the membrane surface is primarily responsible for the high filtration resistance of the sludge cake observed in MBR [14]. The detrimental function of BPC as an important foulant offers new insight into the mechanism of membrane fouling in MBR. However, direct evidence has yet to be found for the actual occurrence of BPC in an MBR system. The chemical nature and the formation and accumulation of BPC in MBR remain to be investigated. 
Fluorescently labelled staining techniques have been used to examine the distributions of microbial cells and EPS in activated sludge [15], aerobic granules [16,17] and biofilms [18] via confocal laser scanning microscopy (CLSM). Kim et al. [19] stained the cells, polysaccharides and proteins in biofilm using different probes for microscopic observation. Chen et al. [20] elucidated the structure of the biofouling layer with CLSM images using a multiple staining scheme for nucleic acids, proteins and $\alpha$ - and $\beta$-d-glucopyranose polysaccharides in the fouling layer. Ferrando et al. [21] was able to characterise membrane fouling with CLSM and to visualise and identify several fouling agents in a sludge mixture. In the experimental study reported herein, CLSM was employed together with a triple staining method to identify the different foulants in the MBR suspension and the sludge fouling layer on the membrane surface. Individual BPC were separated from the biomass and visualised under CLSM. The main chemical composition of the BPC in terms of their polysaccharide, protein and humic content was analysed, and the effect of BPC on MBR fouling was demonstrated.

\section{Materials and Methods}

\subsection{MBR set-up and operation}

A laboratory submerged membrane bioreactor (SMBR) with a working volume of $5 \mathrm{~L}$ was used for the membrane fouling study. A $0.4-\mu \mathrm{m}$ polyethylene hollow-fibre membrane module (surface area $=0.2 \mathrm{~m}^{2}$, Mitsubishi Rayon) was immersed in the SMBR. A pump was used to withdraw the effluent through the membrane at a filtration-to-idle cleaning ratio of 18 min:2 min. Aeration was provided at the bottom of the reactor for continuous membrane cleaning. While the SMBR was operated in constant flux mode, the trans-membrane pressure (TMP) was monitored with a manometer in mmHg. The TMP was increased gradually with 
time from an initial value of about $7 \mathrm{kPa}$ to around $80 \mathrm{kPa}$ before membrane cleaning with a water wash. The other details of the set-up and operation of the SMBR are similar to those previously described $[7,22]$.

The influent to the MBR was glucose-based synthetic wastewater that was prepared according to the basic recipe given in the Environmental Engineering Process Laboratory Manual of the AEESP (2001) [23]. Other chemicals included $\mathrm{NH}_{4} \mathrm{Cl}, \mathrm{KH}_{2} \mathrm{PO}_{4}, \mathrm{NaH}_{2} \mathrm{PO}_{4}$, $\mathrm{CaCl}_{2}, \mathrm{MgCl}_{2}$ and yeast extract. The actual domestic sewage collected from the Stanley Sewage Treatment Works in Hong Kong was dosed into the influent to add $10 \%$ of the organic load. The feeding wastewater had a chemical oxygen demand (COD):N:P ratio of 100:9:3, a total organic carbon (TOC) concentration of around $220 \mathrm{mg} / \mathrm{L}$ and a protein content of about $30 \mathrm{mg} / \mathrm{L}$. The biomass concentration in terms of the suspended sludge (SS) was maintained at a level of around $10 \mathrm{~g} / \mathrm{L}$ throughout the 10 months of the MBR experiment.

\subsection{Sludge samples}

During the MBR operation, both the effluent and the bulk activated sludge (AS) from the MBR suspension were sampled twice a week. For an AS suspension sample of $50 \mathrm{~mL}$, the biomass and the liquid phase were separated by centrifugation at $4000 \mathrm{~g}$ for $15 \mathrm{~min}$. The centrate of the sludge, or the AS supernatant, was collected to analyse its organic content, including its TOC and the concentrations of proteins, polysaccharides and humic substances. The organic content of the MBR effluent was regarded as SMP. The difference in TOC concentration between the AS supernatant and the MBR effluent was assigned to the BPC in the liquid phase of the AS suspension. Subsequently, a portion of the AS supernatant was filtered through a $0.4-\mu \mathrm{m}$ polycarbonate membrane filter (Osmonics). The non-filterable BPC collected on the filter were then dissolved in $20 \mathrm{~mL}$ of warm deionised water, and the organic matter in the solution was analysed for its protein, polysaccharide and humic content. 
When the membrane in the SMBR was severely fouled, the cake sludge (CS) layer was scraped off the membrane surface using a spatula. The recovered CS was re-suspended and dispersed by stirring it into a $0.05 \% \mathrm{NaCl}$ solution to a $\mathrm{SS}$ concentration of around $10 \mathrm{~g} / \mathrm{L}$. The CS suspension of $50 \mathrm{~mL}$ was then centrifuged at $4000 \mathrm{~g}$ for $15 \mathrm{~min}$, and the centrate was collected for organic analysis. A portion of the CS supernatant was then filtered through a 0.4- $\mu \mathrm{m}$ polycarbonate membrane filter (Osmonics). The difference in TOC between the CS supernatant and its filtrate was the BPC in the CS. The non-filterable BPC on the filter was dissolved in $20 \mathrm{~mL}$ of warm water and analysed for its composition of proteins, polysaccharides and humic substances.

The biomass of the MBR sludge was also analysed for its EPS. After sludge-water separation by centrifugation, the sludge pellet in the centrifugal tube was re-suspended using a vortex mixer (Maxi Mix II, Thermolyne) in $0.05 \% \mathrm{NaCl}$ solution to the original volume (50 $\mathrm{mL}$ ) of the sludge sample. Following a heat extraction method [24], the sludge suspension was heated to $50^{\circ} \mathrm{C}$ for $25 \mathrm{~min}$ in a water bath. A lower temperature was used to minimise cell breakage and lysis during EPS extraction [25]. The sludge mixture was then centrifuged at $4000 \mathrm{~g}$ for $15 \mathrm{~min}$, and the centrate was regarded as the EPS extract of the sludge.

\subsection{Analytical analysis}

Each of the liquid samples, including the MBR effluent, the AS supernatant, the CS supernatant and filtrate, and the EPS extract of the sludge, was analysed for its concentrations of TOC, proteins, polysaccharides and humic substances. The TOC was measured with a TOC analyser (TOC-5000A, Shimadzu). The protein and humic content was determined via an UV/VIS spectrophotometer (Lambda 25, Perkin Elmer) following the modified Lowry method [26] and using albumin bovine (Sigma) and humic acids (Fluka) as the standards, respectively. The polysaccharide content was measured according to the phenol method [27] 
using glucose as the standard. In addition, the influent COD and the SS of the sludge suspension were measured in accordance with Standard Methods [28].

\subsection{Visualisation and examination of biopolymer clusters (BPC)}

CLSM was employed to identify the BPC in the liquid phase of the SMBR sludge samples. For sample preparation, the biomass and liquid of the sludge (AS or CS) suspension were separated by sedimentation for $12 \mathrm{hrs}$ at $4^{\circ} \mathrm{C}$ in a refrigerator. The supernatant was then

filtered gently through a $0.4-\mu \mathrm{m}$ black polycarbonate membrane $(25 \mathrm{~mm}$, Osmonics), and the materials collected on the filter were stained before microscopic observation $[7,17,29]$. Three probes were applied collectively: SYTO9 $(25 \mu \mathrm{M}$, Molecular Probes, Eugene, OR) to target all bacterial cells, ConA-TRITC (250 mg/L, Molecular Probes, Eugene, OR) to target the polysaccharides with D-glucose or D-mannose, and NanoOrange (10 mg/L, Molecular Probes, Eugene, OR) to target the proteins. The filter was cut into $5 \times 5 \mathrm{~mm}^{2}$ pieces that were then placed and stained in slide wells. The samples were incubated in a conical centrifuge tube (50-mL, Corning, Corning, NY) at room temperature for $10 \mathrm{~min}$. After staining, all of the slide wells were washed gently three times with a phosphate buffer saline (PBS) solution to remove any unbound probes.

The stained filter was observed for BPC and cells with a CLSM (LSM Pascal, Zeiss, Thornwood, NY). Probe detection was carried out using an optical filter set that consisted of an HFT488 nm/545 $\mathrm{nm}$ beam splitter with a $488 \mathrm{~nm}$ laser beam. An NFT488 $\mathrm{nm}$ beam splitter was used together with a BP505-530 nm bandpass filter for the bacteria stained by SYTO9, and its combination with a setting of $543 \mathrm{~nm} / \mathrm{NFT} 545 / \mathrm{LP} 560$ was used for the polysaccharides stained by ConA-TRITC [7,17]. An NFT488 nm beam splitter and a BP560$585 \mathrm{~nm}$ filter were used for the proteins stained by NanoOrange [30]. Two lenses, 40X/0.85NA (Fluar), were used for the bacterial cells and BPC polysaccharides, with 
10X/0.5NA (Fluar) used for the BPC polysaccharides. The stained BPC particles in the CLSM image were sized and counted using image analysis software (AnalySIS, 3.1, Soft Imaging System, Olympus, Germany). More than 50 view-fields were analysed for each sample to obtain a size distribution of BPC larger than $2.5 \mu \mathrm{m}$.

The biomass of the sludge (AS and CS) samples was also observed with CLSM. The AS suspension $(\sim 10 \mathrm{~g} / \mathrm{L})$ of $1 \mathrm{~mL}$ was filtered to form a thin sludge layer on a $0.4-\mu \mathrm{m}$ black polycarbonate membrane filter (25 mm, Osmonics) for microscopic observation. Afterwards, an additional $20 \mathrm{~mL}$ of the AS supernatant was filtered through the filter. The biomass and BPC on the filter were stained and examined under the CLSM following the procedure previously described.

\section{Results and Discussion}

\subsection{CLSM visualisation of the biopolymer clusters in the SMBR sludge}

The supernatant of the MBR sludge mixture had a consistently higher organic concentration than did that of the permeate effluent (Fig. 1), which agrees well with previous findings $[7,31,32]$. On average, the organic content measured as TOC in the MBR effluent accounted for less than $70 \%$ of the organic content in the liquid phase of the bulk sludge. The organic residue in the effluent was mainly that of SMP [31-33]. The reduction in organic concentration from the MBR sludge supernatant to the effluent indicates a considerable retention of organic solutes by membrane filtration. The organic substances other than SMP in the MBR suspension are specified as BPC [13], which are apparently larger than SMP and unable to pass through the membrane with the permeate.

With CLSM after staining, the individual BPC collected on the filters from the supernatants of the MBR sludge mixture and the cake sludge could be directly visualised. 
Both the microbial cells and the organic macromolecules could be well recognised and differentiated thanks to the triple fluorescent staining technique. The CLSM photos show a large number of BPC particles in the liquid phase of the MBR sludge (Fig. 2a). A few bacterial cells, which appear as green dots in the photos, were also found. Some bacteria were embedded within the clusters; however, there were no typical microbial aggregates left in the AS or CS supernatant samples (Figs. $2 a$ and $2 b$ ). The CLSM images provide clear evidence that BPC are neither biomass flocs nor microbial EPS; instead, they are a group of free and large organic clusters that are present in the liquid phase of the AS mixture and in the CS layer of the MBR.

Proteins, polysaccharides and humic substances are the main components of microbial products $[34,35]$. Upon fluorescent staining, both polysaccharides (red in Figs. $2 \mathrm{a}_{1}$ and $2 \mathrm{~b}_{1}$ ) and proteins (orange in Figs. $2 \mathrm{a}_{2}, 2 \mathrm{a}_{3}, 2 \mathrm{~b}_{2}$ and $2 \mathrm{~b}_{3}$ ) became visible as BPC components. The CLSM images indicate that BPC are clusters of such organic macromolecules as polysaccharides and proteins. It has been suggested that during MBR filtration BPC are formed from the affinitive clustering of SMP and free EPS on the membrane surface [14]. However, unlike the EPS that are associated with microbial cells, BPC are independent of biomass and can be readily separated from the sludge through configuration or sedimentation. Different from SMP, which are primarily filterable organic substances, BPC are much larger organic solutes (Fig. 2). The BPC in the supernatant of the AS suspension ranged in size from 2.5 to about $40 \mu \mathrm{m}$, and those in the CS supernatant ranged from 2.5 to more than $60 \mu \mathrm{m}$ (Fig. 3). For the same amount of biomass, the CS removed from the fouled membrane surface contained more abundant and larger BPC than did the MBR bulk sludge. It should be noted that some BPC, particularly the large ones, may have been broken into small BPC during the pre-treatment for CLSM observation. As a result, the BPC abundance and size distribution may have been under-represented by the CLSM images. 


\subsection{Chemical analysis of biopolymer clusters}

The cake-layer sludge had a BPC content of more than $16.8 \mathrm{mg} \mathrm{TOC} / \mathrm{g}$ SS with a higher protein fraction of around 50\%, a lower polysaccharide fraction of about $30 \%$ and a humic fraction of $20 \%$ (Figs. $4 \mathrm{a}$ and $4 \mathrm{~b}$ ). In comparison, the MBR bulk sludge had a lower $\mathrm{BPC}$ content of about $0.4 \mathrm{mg} \mathrm{TOC} / \mathrm{g}$ SS with protein, polysaccharide and humic proportions of around $40 \%, 45 \%$ and $15 \%$, respectively. The BPC contained less humic materials than did the EPS of the MBR sludge, which had protein, polysaccharide and humic content of around $40 \%, 20 \%$ and 40\%, respectively (Fig. 4). This is most likely due to the firm attachment of humic matter to the surface of the bacterial cells and hence to less release of humic materials into the liquid phase of the sludge suspension [35].

The ratio of proteins/polysaccharides (PN/PS) in the CS BPC (1.45) was higher than that in the bulk sludge BPC (0.80). According to Lee et al. [33] and Sponza [36], a higher PN/PS ratio induces a higher level of hydrophobicity. In relation to their hydrophobicity and surface charge, the affinity between proteins and sludge flocs should generally be greater than that between polysaccharides and flocs [37]. This would help to explain why a greater amount of protein was found in the BPC within the CS layer, although a higher amount of polysaccharides was found in the BPC in the AS suspension.

In relation to the biomass content, the SMP in the MBR effluent was about $0.2 \mathrm{mg}$ $\mathrm{TOC} / \mathrm{g}$ SS on average, with a protein fraction of around $25 \%$, a polysaccharide fraction of about $40 \%$ and a humic fraction of more than $35 \%$ (Fig. 4). The AS supernatant contained both SMP and BPC, for an average organic content of about $0.6 \mathrm{mg}$ TOC/g SS (Fig. 4). There were greater proportions of polysaccharides and proteins and a small fraction of humic substances in the AS supernatant than in the effluent SMP. This comparison between the AS supernatant and the effluent SMP suggests that polysaccharides and proteins are more likely to accumulate in the MBR bulk sludge to form BPC solutes, whereas humic matter is less 
prone to accumulation in the MBR suspension. It is expected that the hydrophobic interactions and cationic bridging between the humic-like substances and the biomass sludge induce the sorption of humic materials onto the solid phase of the sludge [38]. In terms of their chemical composition, BPC are closer to SMP than to EPS (Fig. 4), which suggests that SMP are probably the main precursors of the BPC in the MBR system.

\subsection{BPC formation and its role in membrane fouling}

SMBR provides a unique environment for the formation and accumulation of BPC in the bioreactor. Unlike the conventional activated sludge process, which relies on sedimentation for biomass-effluent separation, the MBR utilises membrane filtration for this purpose. The filtration of a high concentration of sludge results in a layer of sludge attachment, even if it is temporary and reversible, on the membrane surface. A number of studies have shown that the sludge deposition on the membrane surface, rather than pore clogging, is the main cause of membrane fouling $[3,5,7,9]$. The sludge fouling layer on the membrane functions as a porous medium for filtration with an adsorption or affinity capability to retain SMP and colloidal organics $[6,7]$. Because of the effect of filtration, SMP and loose EPS of different sizes concentrate in the sludge layer where they have a greater chance of attaching to each other to form BPC. The forces for SMP aggregation and clustering may include hydrogen bonding, hydrophobic interaction, van der Waals forces and electrostatic attractions [39]. A fluid shear force is maintained by aeration in the SMBR to attenuate the sludge layer growth on the membrane surface. With the turbulent shear, the sludge attached to the membrane, together with the SMP and BPC, is scoured continuously back to the sludge suspension.

Both the SMP and the BPC already in the sludge suspension are forced by filtration onto the membrane surface. Owing to their large sizes, the BPC have a greater likelihood of 
being retained in the sludge layer than do the SMP. It is apparent that the dynamic and cycling process of the sludge and the BPC attachment to and detachment from the membrane surface facilitates the clustering and growth of BPC in the SMBR system. However, the large BPC in the sludge suspension are also subject to breakage by the fluid shear, and thus some BPC may be retained in the fouling layer as stagnant foulants. Hence, it is reasonable to believe that SMP are the main precursors of BPC and the sludge cake layer functions as the sink of BPC in the MBR.

The BPC formation phenomenon was demonstrated by a filtration test with CLSM observation. Filtration of the MBR bulk sludge formed a thin sludge layer on the membrane filter, and a few large BPC within the sludge layer were found (Fig. 5a). The sludge layer was constructed with biomass flocs (green). The bound EPS (yellow) and some small BPC (red) also were visible in the sludge layer. Following filtration of the AS supernatant obtained after sludge sedimentation, a remarkable increase in the number and size of BPC was observed within the sludge layer (Fig. 5b). BPC (red) were entrapped and incorporated into the sludge layer. Due to their high affinity, BPC appeared to attach with each other to form large-sized clusters that filled the pores in the sludge layer. Some of the BPC formed in the sludge layer were larger than those originally present in the AS supernatant. With the formation and retention of large BPC in the sludge layer, its filtration resistance increased significantly. The apparent formation and growth of BPC in the sludge cake resulted not only from their interception, but also from the aggregation and clustering of SMP and small BPC within the sludge layer. In fact, the new sludge layer with the BPC retained appears to be rather similar to the CS removed from the fouled membrane surface (Fig. 5c).

Based on the CLSM observations, it can be concluded that membrane filtration of MBR sludge facilitates the formation and accumulation of BPC in the MBR system. Meanwhile, BPC behave most likely as a glue for the deposition of biomass on the membrane 
surface to form a sticky and impermeable sludge cake. As previously reported $[13,14]$, the sludge cake layer on the fouled membrane surface has much greater specific filtration resistance than does the simple sludge cake formed by the dewatering filtration of the MBR bulk sludge. Therefore, BPC are important foulants in the liquid phase of the sludge mixture. The crucial role played by BPC in MBR membrane fouling should not be overlooked.

\section{Conclusions}

- $\mathrm{BPC}$ are a pool of large organic solutes with a size distribution of up to $60 \mu \mathrm{m}$ in the liquid phase of MBR sludge. Using a triple fluorescent staining protocol, i.e., SYTO9 for microbial cells, ConA-TRITC lectin for polysaccharides and NanoOrange for proteins, the presence of BPC can be well visualised under CLSM. It was evidenced in the experiment reported here that $\mathrm{BPC}$ are important MBR foulants that are much larger than SMP and independent of sludge flocs and EPS.

- The CS on the fouled membrane had a BPC content of $16.8 \mathrm{mg}$ TOC/g SS, which is much higher than that of the MBR bulk sludge at $0.4 \mathrm{mg}$ TOC/g SS. Compared with EPS, BPC contain more polysaccharides and proteins and less humic substances. SMP are believed to be the main precursors of the BPC formed in the MBR.

- A filtration test with CLSM observation demonstrated that BPC are formed by the adsorption and affinity clustering of SMP and colloidal organic matter within the sludge layer deposited on the membrane. The BPC behave as a glue to facilitate the formation and growth of an impermeable sludge cake on the membrane surface, thus resulting in serious MBR fouling. An effective operating strategy needs to be developed to minimise the formation and accumulation of BPC in MBR systems. 


\section{Acknowledgments}

This research was supported by grants N-HKU737/04 and HKU7144/E07 from the Research Grants Council (RGC) of the Hong Kong SAR Government and funding from the Outstanding Young Researcher Award of The University of Hong Kong, China. The instruction of Dr T. Zhang on CLSM procedures and the technical assistance of Mr Keith C. H. Wong are greatly appreciated.

\section{Reference:}

[1] T. Stephenson, S.J. Judd, B. Jefferson, K. Brindle, Membrane Bioreactors for Wastewater Treatment, IWA Publishing, London, 2000.

[2] B. Lesjean, S. Rosenberger, J.C. Schrotter, Membrane-aided biological wastewater treatment—an overview of applied systems, Membr. Technol. 8 (2004), 5-10.

[3] B.D. Cho, A.G. Fane, Fouling transients in nominally sub-critical flux operation of a membrane bioreactor, J. Membr. Sci. 209 (2002), 391-403.

[4] S.J. Judd and B. Jefferson, Membrane for Industrial Wastewater Recovery and Re-use. Elsevier, Oxford, UK, 2003.

[5] J.M. Lee, W.Y. Ahn, C.H. Lee, Comparison of the filtration characteristics between attached and suspended growth microorganisms in submerged membrane bioreactor, Water Res. 35 (2001), 2435-2445.

[6] I.-S. Chang, P. Le-Clech, B. Jefferson, S.J. Judd, Membrane fouling in membrane bioreactors for wastewater treatment, J. Environ. Eng.-ASCE. 128 (11) (2002), 10181029.

[7] H.P. Chu, X.Y. Li, Membrane fouling in a membrane bioreactor (MBR): sludge cake formation and fouling characteristics, Biotechnol. Bioeng. 90 (2005), 323-331. 
[8] A. Ramesh, D.J. Lee, M.L. Wang, J. Hsu, R. Juang, K. Hwang, J. Liu, S. Tseng, Biofouling in membrane bioreactor, Sep. Sci. Technol. 41 (2006), 1345-1370.

[9] P. Le-Clech, B. Jefferson, I.S. Chang, S.J. Judd, Critical flux determination by the fluxstep method in a submerged membrane bioreactor, J. Membr. Sci. 227 (2003), 81-93.

[10] P. Le-Clech, V. Chen, A.G. Fane, Fouling in membrane bioreactors used in wastewater treatment—A review, J. Membr. Sci. 284 (2006), 17-53.

[11] K. Kimura, N. Yamato, H. Yamamura, Y. Watanabe, Membrane fouling in pilot-scale membrane bioreactors (MBRs) treating municipal wastewater, Environ. Sci. Technol. 39 (2005), 6293-6299.

[12] F. Meng, H. Zhang, F. Yang, S. Zhang, Y. Li, X. Zhang, Identification of activated sludge properties affecting membrane fouling in submerged membrane bioreactors, Sep. Purif. Technol. 51 (2006), 95-103.

[13] X.M. Wang, X.Y. Li, Accumulation of biopolymer clusters in a submerged membrane bioreactor and its effect on membrane fouling, Water Res. 42 (2008), 855-862.

[14] X.M. Wang, X.Y. Li, X. Huang, Membrane fouling in a submerged membrane bioreactor (SMBR): Characterisation of the sludge cake and its high filtration resistance, Sep. Purif. Technol. 52 (2007), 439-445.

[15] C. Lopez, M.N. Pons, E. Morgenroth, Evaluation of microscopic techniques (epifluorescence microscopy, CLSM, TPE-LSM) as a basis for the quantitative image analysis of activated sludge, Water Res. 39 (2006), 456-468.

[16] M.Y. Chen, D.J. Lee, J.H. Tay, Distribution of extracellular polymeric substances in aerobic granules, Appl. Microbiol. Biotechnol. 73 (2007), 1463-1469.

[17] S.F. Yang, X.Y. Li, H.Q. Yu, Formation and characterisation of fungal and bacterial granules under different feeding alkalinity and $\mathrm{pH}$ conditions, Process Biochem. 43 (2008), 8-14. 
[18] M. Yun, K. Yeon, J. Park, C. Lee, J. Chun, D.J. Lim, Characterization of biofilm structure and its effect on membrane permeability in MBR for dye wastewater treatment, Water Res. 40 (2006), 45-52.

[19] H.Y. Kim, K.M. Yeon, C.H. Lee, S.H. Lee, T. Swaminathan, Bio-film structure and extracellular polymeric substances in low and high oxygen bioreactors, Sep. Sci. Technol. 41 (2006), 1213-1230.

[20] M.Y. Chen, D.J. Lee, J.Y. Lai, Excellular polymeric substances in fouling layer, Sep. Sci. Technol. 41 (2006), 1345-1370.

[21] M. Ferrando, A. Rozek, M. Zator, F. Lopez, C. Guell, An approach to membrane fouling characterization by confocal scanning laser microscopy, J. Membr. Sci. 250 (2005), 283-293.

[22] X.Y. Li, X.M. Wang, Modelling of membrane fouling in a submerged membrane bioreactor, J. Membr. Sci. 278 (2006), 151-161.

[23] AEESP, Environmental Engineering Process Laboratory Manual, Association of Environmental Engineering and Science Professors, Champaign, IL, 2001.

[24] J.W. Morgan, C.F. Forster, L. Evison, A comparative-study of the nature of biopolymers extracted from anaerobic and activated sludges, Water Res. 24 (1990), 743-750.

[25] X.Y. Li, S.F. Yang, Influence of extracellular polymeric substances (EPS) on the flocculation, sedimentation and dewaterability of activated sludge, Water Res. 41 (2007), $1022-1030$.

[26] B. Frolund, T. Griebe, P.H. Nielsen, Enzymatic-activity in the activated-sludge floc matrix, Appl. Microbiol. Biotechnol. 43 (1995), 755-761.

[27] P. Gerhardt, R.G.E. Murray, W.A. Wood, N.R. Krieg, Methods for General and Molecular Bacteriology, American Society for Microbiology, Washington, DC, 1994. 
[28] APHA-AWWA-WEF, Standard Methods for the Examination of Water and Wastewater, 20th ed. American Public Health Association/American Water Works Association/Water Environment Federation, Washington, DC, 1998.

[29] T. Zhang, H.H.P. Fang, Quantification of extracellular polymeric substances in biofilms by confocal laser scanning microscopy, Biotechnol. Lett. 23 (2001), 405-409.

[30] J.L. Alonso, S. Mascellaro, M.A. María, J. Hernández, Double-staining method for differentiation of morphological changes and membrane integrity of Campylobacter coli cells, Appl. Environ. Microbiol. 68 (2002), 5151-5154.

[31] H. S. Shin, S. T. Kang, Characteristics and fates of soluble microbial products in ceramic membrane bioreactor at various sludge retention times, Water Res. 37 (2003), 121-127.

[32] S. Liang, C. Liu, L. Song, Soluble microbial products in membrane bioreactor operation: behaviors, characteristics, and fouling potential, Water Res. 41 (2007), 95-101.

[33] W. Lee, S. Kang, H. Shin, Sludge characteristics and their contribution to microfiltration in submerged membrane bioreactors, J. Membr. Sci. 216 (2003), 217-227.

[34] B. Frolund, R. Palmgren, K. Keiding, P.H. Nielsen, Extraction of extracellular polymers from activated sludge using a cation exchange, Water Res. 30 (1996), 1749-1758.

[35] C.S. Laspidou, B.E. Rittmann, A unified theory for extracellular polymeric substances, soluble microbial products, and active and inert biomass, Water Res. 36 (2002), 27112720.

[36] D.T. Sponza, Investigation of extracellular polymeric substances (EPS) and physicochemical properties of different activated sludge flocs under steady-state conditions, Enzyme Microb. Technol. 32 (2003), 375-385.

[37] D. Renard, L. Lavenant, C. Sanchez, Y. Hemar, D. Horne, Heat-induced flocculation of microparticulated whey proteins (MWP); consequences for mixed gels made of MWP and $\beta$-lactoglobulin, Coll. Surf. B. 24 (2002), 73-85. 
[38] M. Esparza-Soto, P. Westerhoff, Biosorption of humic and fulvic acids to live activated sludge biomass, Water Res. 37 (2003), 2301-2310.

[39] W. Stumm, J.J. Morgan, Aquatic Chemistry: Chemical Equilibria and Rates in Natural Waters, $3^{\text {rd }}$ edition, Wiley, New York, 1995. 


\section{Figure captions:}

Fig. 1. Organic concentrations in the supernatant of the sludge suspension and the effluent throughout the MBR operation.

Fig. 2. CLSM images of biopolymer clusters in the supernatants of $\left(a_{1}-a_{3}\right)$ the MBR bulk sludge and $\left(\mathrm{b}_{1}-\mathrm{b}_{3}\right)$ cake sludge: $\left(\mathrm{a}_{1}\right)$ and $\left(\mathrm{b}_{1}\right)$ polysaccharides (red) and cells (green); $\left(\mathrm{a}_{2}\right)$ and $\left(b_{2}\right)$ proteins (orange); $\left(a_{3}\right)$ and $\left(b_{3}\right)$ proteins (orange) and cells (green) (scale bar $=50$ $\mu \mathrm{m})$.

Fig. 3. The size distribution of BPC in the supernatants of (a) the MBR bulk sludge and (b) the cake sludge from the analysis of the CLSM images.

Fig. 4. (a) The biomass-based organic content of microbial EPS, BPC in cake sludge, BPC in bulk activated sludge, SMP in the effluent and the organic matter in the AS supernatant from the MBR; (b) the proportions of polysaccharides, proteins and humic substances in the corresponding organic samples indicated.

Fig. 5. CLSM images of (a) the bulk activated sludge layer, (b) the bulk AS layer with BPC after filtration of additional AS supernatant and (c) the cake sludge collected from the membrane surface of the MBR. Subscripts 1, 2 and 3 denote the upper, middle and lower positions of the sludge layers on the filters for CLSM observations of bacteria (green) and polysaccharides (red) (scale bar $=10 \mu \mathrm{m})$. 


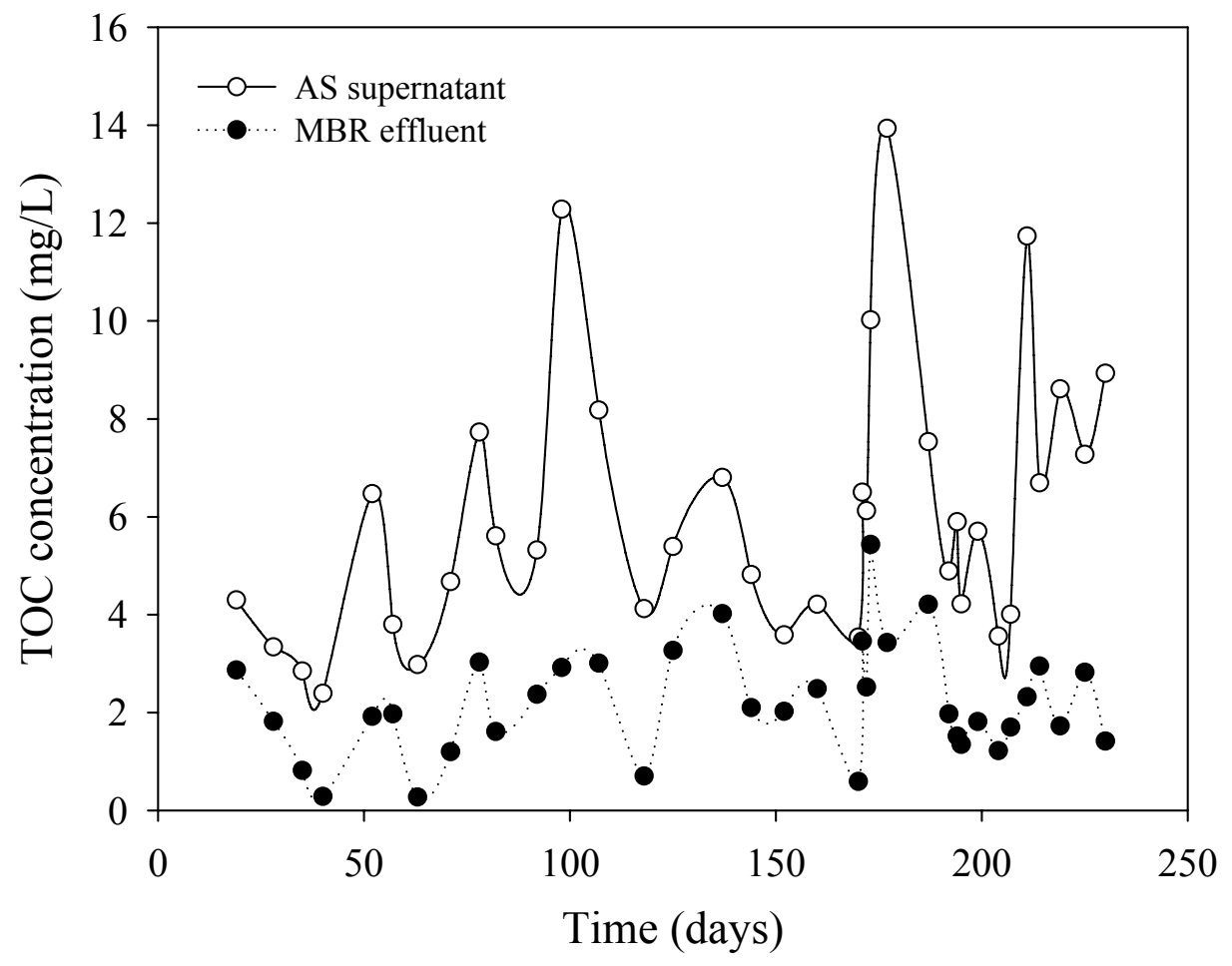

Fig. 1. Organic concentrations in the supernatant of the sludge suspension and the effluent throughout the MBR operation. 

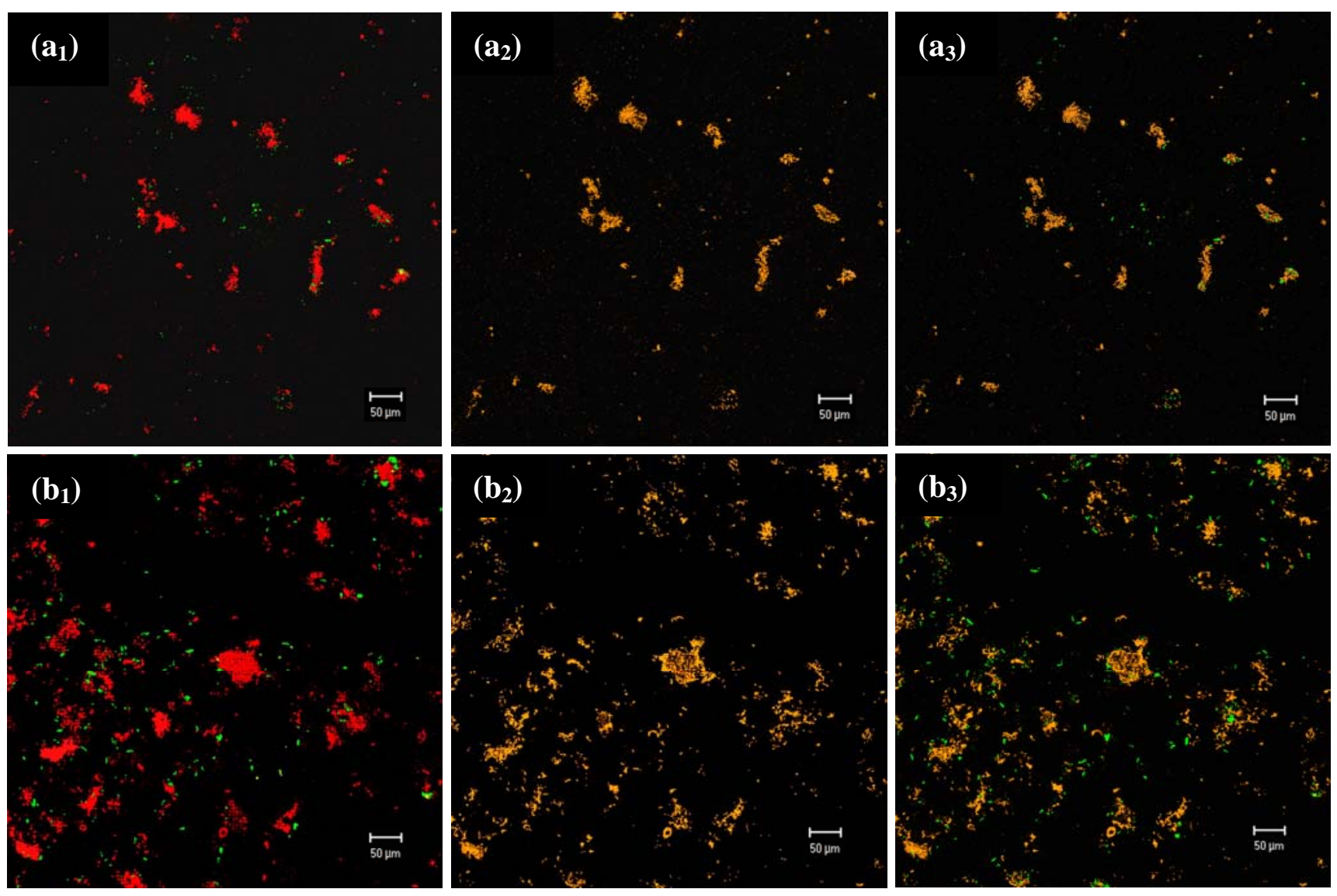

Fig. 2. CLSM images of biopolymer clusters in the supernatants of $\left(a_{1}-a_{3}\right)$ the MBR bulk sludge and $\left(\mathrm{b}_{1}-\mathrm{b}_{3}\right)$ cake sludge: $\left(\mathrm{a}_{1}\right)$ and $\left(\mathrm{b}_{1}\right)$ polysaccharides (red) and cells (green); $\left(\mathrm{a}_{2}\right)$ and $\left(b_{2}\right)$ proteins (orange); $\left(a_{3}\right)$ and $\left(b_{3}\right)$ proteins (orange) and cells (green) (scale bar $=50$ $\mu \mathrm{m})$. 

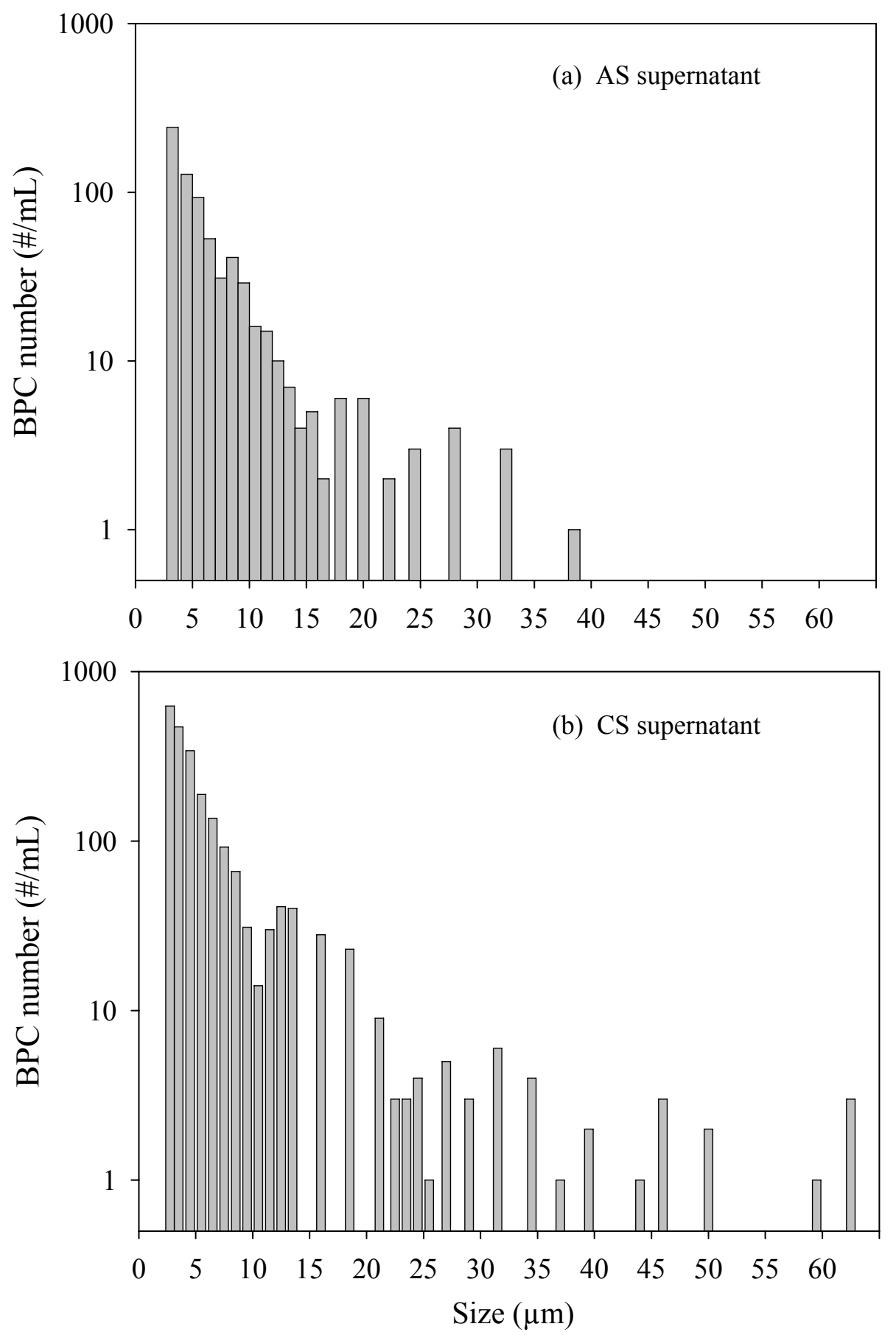

Fig. 3. The size distribution of BPC in the supernatants of (a) the MBR bulk sludge and (b) the cake sludge from the analysis of the CLSM images. 

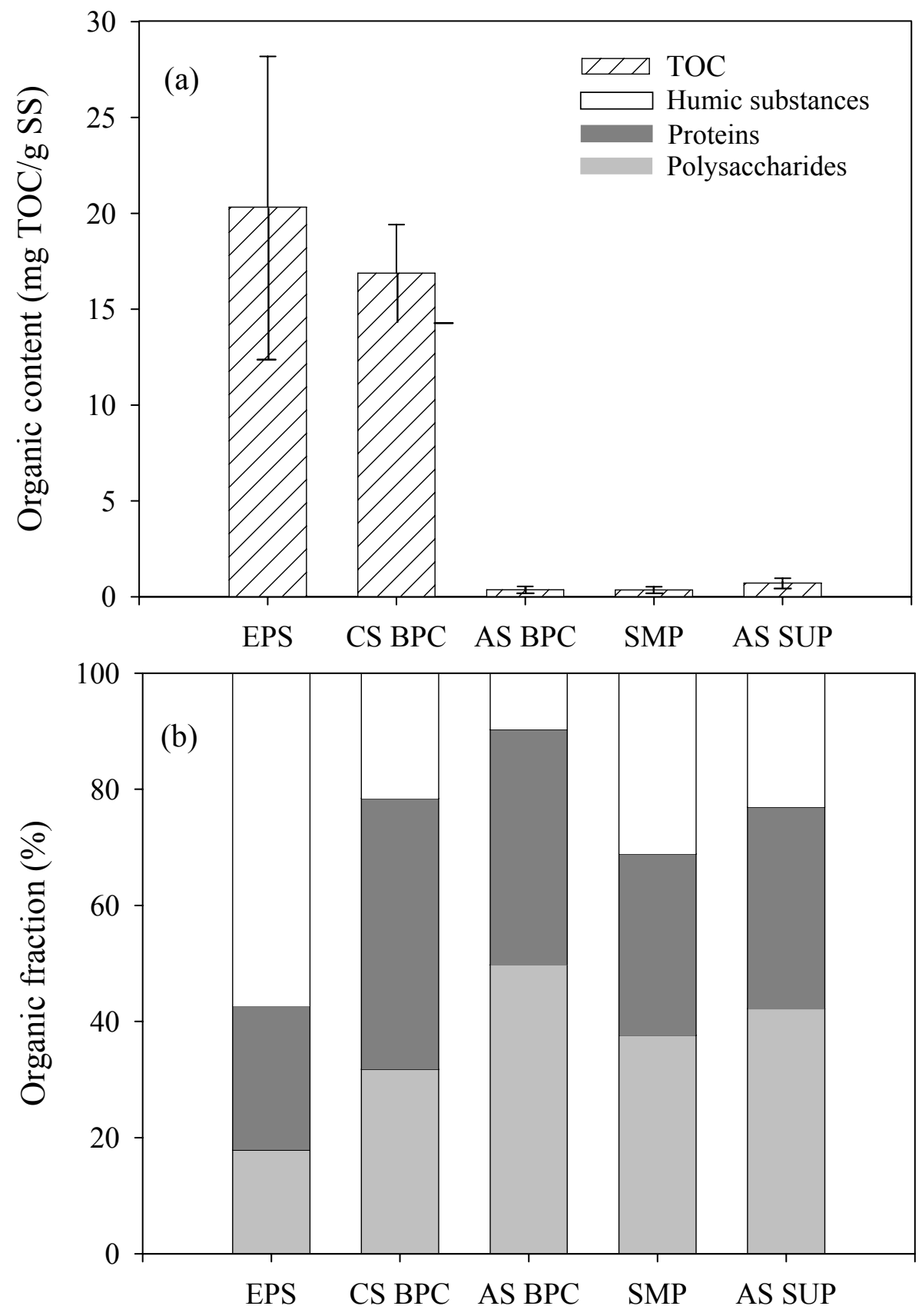

Fig. 4. (a) The biomass-based organic content of microbial EPS, BPC in cake sludge, BPC in bulk activated sludge, SMP in the effluent and the organic matter in the AS supernatant from the MBR; (b) the proportions of polysaccharides, proteins and humic substances in the corresponding organic samples indicated. 


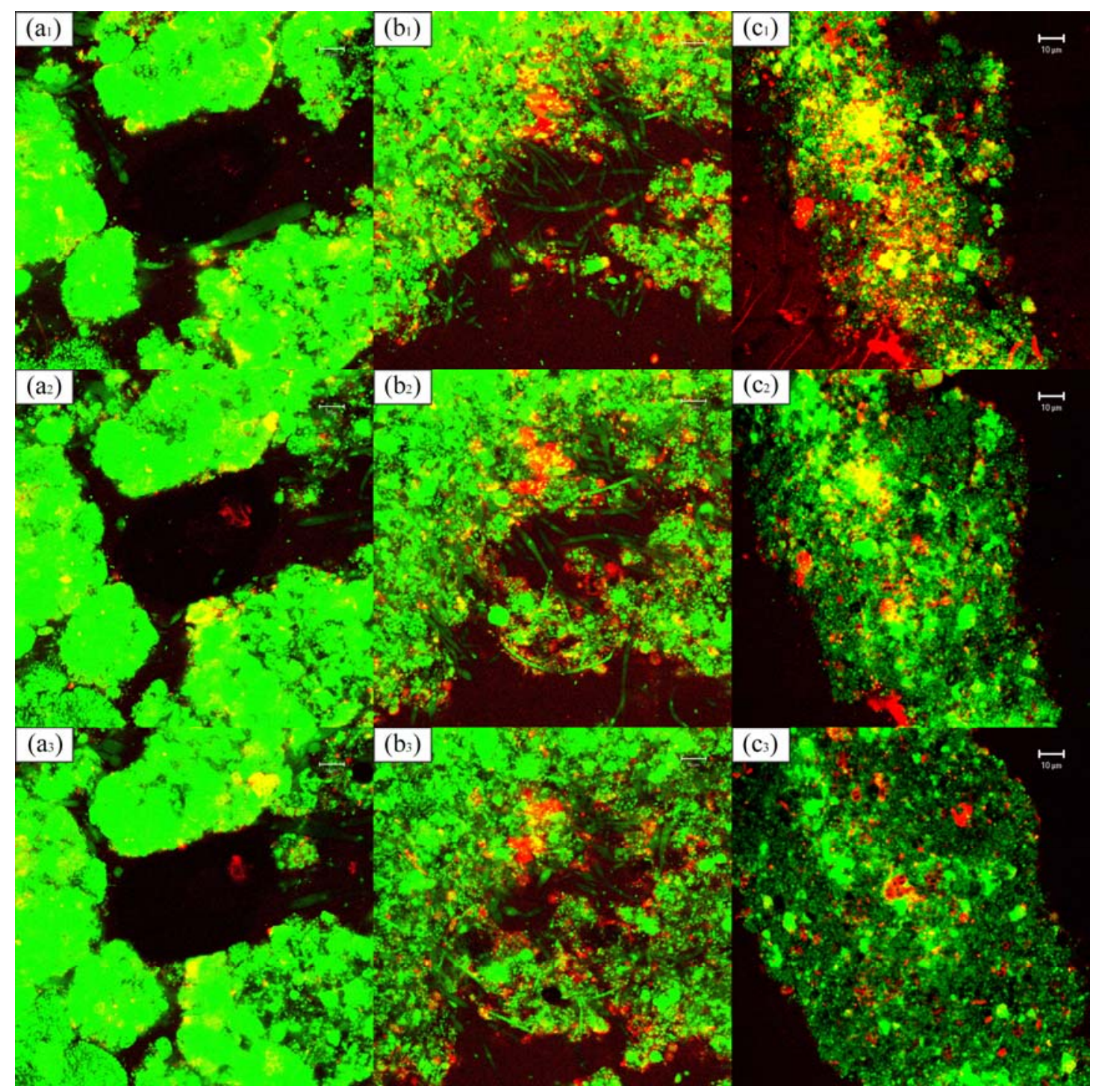

Fig. 5. CLSM images of (a) the bulk activated sludge layer, (b) the bulk AS layer with BPC after filtration of additional AS supernatant and (c) the cake sludge collected from the membrane surface of the MBR. Subscripts 1, 2 and 3 denote the upper, middle and lower positions of the sludge layers on the filters for CLSM observations of bacteria (green) and polysaccharides (red) (scale bar $=10 \mu \mathrm{m})$. 